\title{
Helicobacter pilory en niños con enfermedad celiaca. Hallazgos epidemiológicos y clínicos
}

\author{
Helicobacter pylori in children with celiac disease. \\ Epidemiological and clinical findings
}

\author{
Eduardo Couchonal', Bernardita Corrales', Lidia Garcete
}

\section{RESUMEN}

Introducción: En países en desarrollo se describen prevalencias de Helicobacter pylory (HP) de hasta $80 \%$. Su impacto en los enfermos celiacos es controversial, pero existen indicios que podría agravar la condición celiaca. No se ha confirmado la asociación entre ambas. En nuestro medio, factores socio ambientales pudieran favorecer una elevada prevalencia del HP. Objetivos: Conocer la prevalencia de infección por $\mathrm{HP}$ en una población pediátrica portadora de enfermedad celiaca (EC). Materiales y Métodos: estudio observacional, retrospectivo de corte transversal revisando fichas clínicas y resultados biópsicos de pacientes sometidos a endoscopia digestiva alta por la sospecha clínica y laboratorial (marcadores serológicos positivos) de enfermedad celiaca en el Departamento de Gastroenterología del Servicio de Pediatría del Hospital de Clínicas de la Facultad de Ciencias Médicas. Resultados: Se incluyeron en el estudio 50 muestras de biopsias gástricas y duodenales. Todos reunieron criterios histológicos de EC, en 9 pacientes (18\%) se observó la presencia de HP. No se encontraron diferencias significativas en aspectos socioambientales y tampoco en cuanto a prevalencia de desnutrición y anemia comparando EC con y sin HP. Llamó la atención la presencia de distensión abdominal como dato significativo en los EC portadores de HP. La gastritis crónica activa fue el hallazgo histológico prevalente $(89 \%)$ en los EC con $\mathrm{HP}+$. Conclusión: La prevalencia de $\mathrm{HP}$ en niños celiacos fue del $18 \%$, inferior al esperado, aunque coincidente con un reporte nacional previo.

Palabras clave: Helicobacter pylory, enfermedad celiaca, prevalencia.

\begin{abstract}
Introduction: In developing countries, Helicobacter pylori (HP) prevalences of up to $80 \%$ have been described. Its impact on celiac patients is controversial, but there are indications that it could aggravate the celiac condition. The association between the two has not been confirmed. In our setting, socio-environmental factors could favor a high prevalence of HP. Objectives: To describe the prevalence of HP infection in a pediatric population with celiac disease (CD). Materials and Methods: this was an observational, retrospective and cross-sectional study reviewing clinical records and biopsy results of patients undergoing upper gastrointestinal endoscopy for clinical and laboratory suspicion (positive serological markers) of celiac disease in the Department of Gastroenterology of the Pediatric Service of the University Hospital. Results: 50 gastric and duodenal biopsy samples were included in the study. All met histological criteria for $C D$, in 9 patients $(18 \%)$ the presence of HP was observed. No significant differences were found in socio-environmental aspects or in terms of prevalence of malnutrition and anemia when comparing CD with and without HP. The presence of abdominal distention is striking as a significant finding in $\mathrm{CD}$ with HP. Active chronic gastritis was the prevalent histological finding (89\%) in CD with HP +. Conclusion: The prevalence of HP in celiac children was 18\%, lower than expected, although it was consistent with a previous national report.
\end{abstract}

Key words: Helicobacter pylori, celiac disease, prevalence.

\footnotetext{
${ }^{1}$ Universidad Nacional de Asunción. Facultad de Ciencias Médicas. Cátedra y Servicio de Pediatría. San Lorenzo, Paraguay.

Correspondencia: Lidia Garcete Correo: lidiga1@hotmail.com

Conflicto de interés: Los autores declaran no poseer conflicto de interés.

Recibido: 26/08/2020 Aceptado: 28/09/2020

Doi: https://doi.org/10.31698/ped.47032020004
} 


\section{INTRODUCCION}

La infección por Helicobacter pylory (HP) es una causa frecuente de infección bacteriana crónica que afecta a la población mundial y a todas las edades, representando un problema de salud en países en desarrollo ${ }^{(1)}$. La colonización depende de la virulencia del germen, la susceptibilidad del huésped y condicionantes socios ambientales. Es preponderante de la transmisión fecal oral, considerándose de riesgo las condiciones socioeconómicas y de higiene, disposición de excretas, agua potable, hacinamiento, mascotas en la casa $^{(2)}$. Se adquiere por lo general durante la infancia y su prevalencia aumenta con la edad. En condiciones sanitarias y nutricionales precarias la prevalencia ya es alta al año de vida, llegando al $80 \%$ al final de la adolescencia ${ }^{(1,2)}$. En niños puede causar enteropatía pierde proteínas, diarrea crónica, síndrome de malabsorción, gastritis crónica no autoinmune y retraso pondo estatural ${ }^{(1,3)}$. El diagnostico puede realizarse por el test de aliento con urea marcada, un método no invasivo confiable $^{(1,4)}$ y por el examen histológico de biopsias gástricas que muestra patrones histológicos de gastritis con predominio linfocitario ${ }^{(4,5)}$. El gold standard es el cultivo microbiológico ${ }^{(2,3)}$.

Por su parte la enfermedad celiaca (EC) es un trastorno inmunitario sistémico de base genética, mediado por el gluten, de distribución mundial, homogénea, que afecta a todas las razas y a cualquier edad, disparada por factores ambientales no del todo dilucidados ${ }^{(6)}$. Su prevalencia oscila entre 1:250 a $1 \%$ de la población ${ }^{(6-8)}$. La presentación clásica o típica en la que predominan los síntomas gastrointestinales es prevalente en la infancia ${ }^{(5,6)}$. Existen formas atípicas, silentes y latentes. El diagnóstico se sustenta en la combinación de historia clínica, serología (anticuerpos específicos y estudio genético), biopsia de mucosa duodenal y respuesta a la retirada del gluten de la dieta ${ }^{(6,7)}$.

Con relación a la asociación entre estas entidades, la EC afecta de manera importante a la población pediátrica, y en los países en desarrollo, la colonización por HP se inicia desde edades tempranas. Aunque la patogénesis de la EC es poco comprendida, la posibilidad de que una infección gástrica crónica capaz de inducir úlceras duodenales pueda influir en las respuestas inflamatorias e inmunes en el intestino delgado $y$, por lo tanto, en el desarrollo y la evolución de la EC, ha generado inquietud ${ }^{(9)}$. No existe confirmación de la asociación entre estas dos entidades, aunque existen aspectos que le dan soporte. Entre ellos estarían la respuesta Th1 predominante observada en ambas y la sospecha de que los cambios en la mucosa gástrica ocasionados por el HP pudieran agravar la condición de los celiacos ${ }^{(9,10-12)}$. Estudios recientes han reportado que los pacientes con gastritis linfocítica por HP son pasibles de tener mayor número de linfocitos intraepiteliales en la mucosa duodenal, y que esto puede revertirse mediante la erradicación del $\mathrm{HP}^{(10)}$. Otros estudios han sugerido una relación causal entre infección por HP y anemia en pacientes con EC. El interés se ha generado ante el reporte de elevada prevalencia de HP en sujetos con anemia refractaria, pues como la EC puede cursar con anemia, esto pudiera constituirse en un factor de riesgo para colonización por $\mathrm{HP}^{(9-12)}$. Desde el punto de vista fisiopatológico, la afectación del estado nutricional en la EC podría asociar hipoclorhidria, modificando el ph gástrico y favoreciendo el desarrollo de infecciones. En la EC, al asociar anormalidades de la histología y la función gástrica, la infección por HP podría influir en el desarrollo y la evolución de la enteropatía al modular las respuestas inflamatorias e inmunitarias en el intestino delgado ${ }^{(0,10)}$.

Además, niños con EC por las alteraciones en el estado nutricional e inmunológico, como en el ecosistema y la barrera mucosa intestinal, podrían ser más susceptibles de ser colonizados por HP, aunque hay controversias al respecto ${ }^{(13-17)}$.

En este contexto el objetivo del presente estudio fue determinar la frecuencia de infección por HP en biopsias gástricas de pacientes con EC y los factores epidemiológicos y características clínicas asociados.

\section{MATERIALES Y MÉTODOS}

Se realizó un estudio observacional, retrospectivo de corte transversal revisando los resultados biópsicos de pacientes de 0 a 18 años de edad, sometidos a 
endoscopia digestiva alta en el Departamento de Gastroenterología del Servicio de Pediatría del Hospital de Clínicas de la FCM. UNA. por sospecha clínica y laboratorial (marcadores positivos) de enfermedad celiaca. Posteriormente se efectuó la búsqueda de las fichas clínicas de los pacientes para completar los datos clínicos de los mismos.

Fueron incluidos los pacientes con diagnóstico confirmado de EC por sospecha clínica, marcadores laboratoriales e histología con criterios de MARSH $(+)$ para EC.

Fueron analizadas las variables edad, sexo, peso, talla, acceso a agua potable, tipo de baño, síntomas principales referidos, hallazgos al examen físico, presencia de anemia, presencia de Helicobacter pylory, hallazgos anatomopatológicos asociados.

El estado nutricional se evaluó con el programa WHO Anthro aplicando los criterios de evaluación nutricional de la OMS en base a puntaje $\mathrm{z}$ Peso/Edad (zP/E), z Peso/Talla (zP/T), z Talla/Edad (zT/E), considerándose eutróficos los puntajes z entre $-1 \mathrm{y}+1$ y desnutrición o sobrepeso, los puntajes por debajo o encima de estos rangos respectivamente.

La presencia de anemia se estableció considerando el valor de Hemoglobina del paciente en comparación con los niveles mínimos establecidos por la Organización Mundial de la salud (OMS). Ginebra: OMS; 2011. Se considero anemia cuando los valores de $\mathrm{Hb}$ fueron menores de $11 \mathrm{~g} / \mathrm{dL}$.

Estudio anatomopatológico. Se extrajeron fragmentos de la mucosa del duodeno y del antro gástrico por biopsias per endoscópicas. Las muestras fueron analizadas en la Cátedra de Anatomía Patológica de la FCM - UNA, y el diagnóstico histológico de enfermedad celiaca se estableció por los criterios de la Sociedad Europea de Gastroenterología, Hepatología y Nutrición Pediátrica, presencia de atrofia parcial o total de las vellosidades intestinales, aumento de la profundidad de las criptas y disminución de la relación vellosidad / cripta, aumento del número de linfocitos intraepiteliales ${ }^{(6)}$.

La positividad para Helicobacter pylory se determinó por la observación directa del gérmen a través del microscopio óptico previa tinción con Giemsa.

Las características de los pacientes se analizaron con estadísticas descriptivas, las variables continuas se expresaron como promedios y DE, y medianas con mínimo y máximo, según la distribución. Los datos fueron procesados utilizando el programa EPI Info. Se recurrió a la prueba exacta de Fisher por el escaso número en el grupo HP positivo. La significancia estadística se fijó en un valor de de $\mathrm{p}<0.05$.

En el aspecto ético el trabajo se realizó bajos las normas de la Declaración de Helsinki.

\section{RESULTADOS}

Se incluyeron en el estudio 50 muestras de biopsias gástricas y duodenales. Se excluyeron 5 muestras que resultaron no satisfactorias para efectuar el diagnóstico de infección por Helicobacter pylory.

El $64 \%(32 / 50)$ eran de sexo femenino. El promedio de edad fue de 5,9 años $\pm 3,9$ con un mínimo de 8 meses y máximo de 15 años.

Todos los pacientes incluidos $(\mathrm{n}=50)$ reunieron los criterios histológicos de MARSH para EC. En 9/50 pacientes $(18 \%)$ se observó la presencia de HP. Los datos demográficos, y algunos aspectos de necesidades básicas como provisión de agua y disposición de excretas en los grupos con HP positivo y negativo, se expresan en la tabla 1.

Tabla 1. Datos demográficos, y algunos aspectos de necesidades básicas insatisfechas en pacientes con enfermedad celiaca con y $\sin \mathrm{HP}$. $\mathrm{N}=50$.

\begin{tabular}{lccc}
\hline & $\begin{array}{c}\mathbf{H P}+ \\
\mathbf{n = 9}\end{array}$ & $\begin{array}{c}\mathbf{H P}- \\
\mathbf{n}=\mathbf{4 1}\end{array}$ & $\mathbf{p}$ \\
\hline Edad (años) & & & $0,27^{1}$ \\
Mediana - Min- Max & $7,5(3-12)$ & $6(1-15)$ & \\
Sexo & $\mathrm{N}(\%)$ & $\mathrm{N}(\%)$ & \\
Femenino & $5(55,5)$ & $25(60.9)$ & $1^{2}$ \\
Provisión de agua & $\mathrm{n}=7$ & $\mathrm{n}=34$ & \\
Agua potable & $7(100)$ & $29(85 \%)$ & $0,56^{2}$ \\
Pozo artesiano & 0 & $5(15 \%)$ & \\
Excretas & $\mathrm{n}=7$ & $\mathrm{n}=34$ & \\
Baño moderno & $6(86)$ & $31(91)$ & $0,54^{2}$ \\
Letrina & $1(14)$ & $3(9)$ & \\
\hline
\end{tabular}

$1=\mathrm{U}$ Mann Whitney $2=$ Test Exacto de Fisher

$\mathrm{HP}+=$ Helicobacter pylory positivo

HP - = Helicobacter pylory negativo 
La sintomatología fue similar en los enfermos celiacos HP positivos o negativos, siendo la más frecuente la diarrea crónica. La proporción de los síntomas se encuentra en la tabla 2.

Tabla 2. Síntomas de los pacientes con EC con y sin infección por H P. N=41.

\begin{tabular}{lccc}
\hline & $\begin{array}{c}\mathbf{H P}+ \\
\mathbf{n}=7\end{array}$ & $\begin{array}{c}\mathbf{H P}- \\
\mathbf{n = 3 4}\end{array}$ & $\mathbf{p}$ \\
\hline Diarrea crónica & $\mathrm{N}(\%)$ & $\mathrm{N}(\%)$ & \\
Falla de medro & $5(71,4)$ & $21(62)$ & $1^{1}$ \\
Dolor abdominal & $2(28,5)$ & $11(32)$ & $1^{1}$ \\
Vómitos & 0 & $3(8,8)$ & $1^{1}$ \\
Otros & $1(14,2)$ & $5(14,7)$ & $1^{1}$ \\
\hline
\end{tabular}

$1=$ Test Exacto de Fisher

$\mathrm{HP}+=$ Helicobacter pylory positivo

HP - = Helicobacter pylory negativo

Se pudo analizar el estado nutricional en 45 participantes y la presencia de anemia en 39. Los resultados de la distribución de acuerdo a HP positivo o negativos se encuentran en la tabla 3 .

Tabla 3. Estado nutricional de los EC con y sin infección por HP. $\mathrm{N}=45$.

\begin{tabular}{lccc}
\hline & $\begin{array}{c}\text { HP }+ \\
\mathbf{n}=\mathbf{8}\end{array}$ & $\begin{array}{c}\text { HP - } \\
\mathbf{n}=37\end{array}$ & $\mathbf{p}$ \\
\hline Eutróficos & $\mathrm{N}(\%)$ & $\mathrm{N}(\%)$ \\
Desnutridos & $6(75 \%)$ & $21(56,7 \%)$ & $0,44^{1}$ \\
Sobrepeso & $2(25 \%)$ & $15(40.5 \%)$ & $0,68{ }^{1}$ \\
Anemia & 0 & 1 & \\
Hb Media DE & $4(59 \%)$ & $20(64,5 \%)$ & $0,36^{1}$ \\
\hline
\end{tabular}

$1=$ Test Exacto de Fisher $2=$ T Student

$\mathrm{HP}+=$ Helicobacter pylory positivo

$\mathrm{HP}-=$ Helicobacter pylory negativo

$\mathrm{Al}$ examen físico, tanto en el grupo de EC portador de HP como en el no portador el principal hallazgo fue la distensión abdominal, En los pacientes sin HP se describió en el $24 \%$ (10/41) casos y en los pacientes con HP en el 75\% (6/8) p=0,01.

Considerando la anatomía patológica, en 78\% de las biopsias gástricas se encontró gastritis, predominando la gastritis no activa y crónica activa. se encontró significancia con HP positivo para los casos de gastritis crónica activa $(p<0,05)$. Ver Tabla 4 .
Tabla 4. Hallazgos anatomopatológicos. N=50.

\begin{tabular}{|c|c|c|c|}
\hline & $\begin{array}{c}\mathrm{HP}+{ }^{*} \\
\mathrm{n}=9\end{array}$ & $\begin{array}{l}\mathrm{HP}-* \\
\mathrm{n}=41\end{array}$ & p \\
\hline \multicolumn{4}{|l|}{ Biopsia gástrica } \\
\hline Normal & $0(0 \%)$ & $11(27 \%)$ & $0.04^{1}$ \\
\hline Gastritis crónica no activa & $1(11 \%)$ & $20(49 \%)$ & $0.02^{1}$ \\
\hline Gastritis crónica activa & $8(89 \%)$ & $6(15 \%)$ & $0.00002^{1}$ \\
\hline Gastritis linfocítica & $0(0 \%)$ & $4(19 \%)$ & 0.21 \\
\hline \multicolumn{4}{|l|}{ Biopsia duodenal } \\
\hline Estadio de Marsh 2 & $0(0 \%)$ & $2(5 \%)$ & $0.33^{1}$ \\
\hline Estadio de Marsh 3 a & $1(11 \%)$ & $1(2 \%)$ & $0.18^{1}$ \\
\hline Estadio de Marsh $3 \mathrm{~b}$ & $3(33 \%)$ & $16(39 \%)$ & $0.39^{1}$ \\
\hline Estadio de Marsh $3 \mathrm{C}$ & $5(56 \%)$ & $22(54 \%)$ & $0.46^{1}$ \\
\hline
\end{tabular}

$1=$ Test Exacto de Fisher

$\mathrm{HP}+=$ Helicobacter pylory positivo

$\mathrm{HP}$ - = Helicobacter pylory negativo

\section{DISCUSIÓN}

Analizando la prevalencia de HP en los niños celiacos estudiados, el porcentaje de $18 \%$ es considerado bajo en relación a la prevalencia esperada según otros estudios, pues de acuerdo a las características socio epidemiológicas de nuestro país la misma esperaríamos se encontrase entre $50 \%$ y $80 \%{ }^{(3)}$.

Existe sin embargo similitud con los resultados de un trabajo nacional de prevalencia de HP en biopsias duodenales de niños sometidos a endoscopia digestiva ${ }^{(18)}$, que reportó positividad para HP en $15 \%$ pacientes (12/80). Al comparar la prevalencia de HP en niños celiacos en nuestra investigación, con dicho trabajo, no existieron diferencias significativas $(\mathrm{p}=$ 0,33 ). Reportes de otras poblaciones tampoco han demostrado diferencias en la prevalencia de HP comparando pacientes celiacos y no celiacos ${ }^{(12,13)}$.

Los aspectos epidemiológicos y medioambientales no demostraron asociación con el hallazgo de HP, a diferencia de lo esperado según la literatura ${ }^{(2)}$, aunque con respecto a este punto se podría considerar como limitante, la reducida población de pacientes con la infección HP, lo que impide extraer conclusiones significativas.

Aunque la sintomatología fue similar en ambos grupos, coincidiendo con otros reportes ${ }^{(10,11,13,19)}$ y siendo esperable, considerando que las manifestaciones clínicas gastrointestinales típicas descriptas para la EC pueden superponerse a las del $\mathrm{HP}$, llamó la atención la distensión abdominal, que 
fue un hallazgo que se asoció de manera significativa a la presencia de HP en niños celiacos.

Considerando el impacto que la desnutrición pudiera tener como factor de vulnerabilidad para la infección por $\mathrm{HP}$, aunque en la población total se observó elevada proporción de desnutrición que pudiera indicar demora en el diagnóstico de EC, no se observó diferencias entre pacientes HP positivos o negativos en el estado nutricional. Este aspecto pudiera clarificarse aumentando el tamaño de la muestra.

El hallazgo de anemia fue similar tanto en pacientes $\mathrm{HP}$ positivos como HP negativos, no coincidiendo con los reportes de una mayor prevalencia de HP en la anemia ${ }^{(1)}$. Se requeriría un mayor número de pacientes para discriminar la implicancia de la misma en estas patologías.

Con relación a los hallazgos histológicos, llama la atención la elevada frecuencia de gastritis en la población de EC estudiada 39/50 (78\%), correspondiendo un alto porcentaje a gastritis crónica no activa 20/41 (49\%) en pacientes celiacos sin presencia de HP, lo que pudiera relacionarse con el estatus nutricional de los pacientes y una eventual enteropatía ambiental. En los pacientes celiacos con infección HP predomino la gastritis crónica activa en $8 / 9(89 \%)$ como es de esperarse considerando la fisiopatología del germen.

Es importante señalar que debido a que en esta investigación se utilizó una metodología retrospectiva no pudo discriminarse a niños que utilizaron antibióticos o antiparasitarios poco tiempo antes, lo que podría haber alterado la observación de los microorganismos. Además, el objetivo principal de las biopsias digestivas fue

\section{REFERENCIAS}

1. Pacifico L, Anania C, Osborn JF, Ferraro F, Chiesa C. Consequences of Helicobacter pylori infection in children. World J Gastroenterol. 2010;16(41):5181-5194. doi: http://dx.doi.org/10.3748/wjg.v16.i41.5181 efectuar el diagnóstico de EC, hecho que pudiera afectar la eficacia diagnóstica para el HP. Son necesarios estudios prospectivos que permitan mayor fiabilidad en la determinación de la prevalencia de EC e infección por HP en niños y su posible impacto en su calidad de vida.

\section{CONCLUSIONES}

La prevalencia de HP en niños celiacos fue del 18\%, similar a un estudio local e inferior al esperado para la región.

No se reportaron diferencias relacionadas con factores socio epidemiológicos.

Llamo la atención la presencia de distensión abdominal como dato significativo en los EC portadores de HP.

Aunque no se observó asociación entre deterioro nutricional en los enfermos celiacos y presencia o no de infección HP, por el tamaño de la muestra no es posible concluir el impacto real de la afectación nutricional.

Llamo la atención la elevada frecuencia de gastritis crónica en la histología en la población de EC estudiada, predominando la gastritis crónica activa en los enfermos celiacos portadores de HP y la gastritis crónica no activa en los pacientes HP negativos.

Como hallazgos derivados, en la población general de enfermos celiacos, señalamos la alta prevalencia de desnutrición moderada/severa, talla baja y anemia, que podría estar relacionada con un diagnóstico tardío de la enfermedad celiaca.

2. Ertem D. Clinical practice: Helicobacter pylori infection in childhood. Eur J Pediatr. 2013;172(11):1427-1434. doi: http://dx.doi.org/10.1007/s00431-012-1823-4 
3. Spee LA, Madderom MB, Pijpers M, van Leeuwen $Y$, Berger MY. Association between helicobacter pylori and gastrointestinal symptoms in children. Pediatrics. 2010;125(3):e651-669. doi: http://dx.doi.org/10.1542/pe ds.2010-0941

4. Guarner J, Kalach N, Elitsur Y, Koletzko S. Helicobacter pylori diagnostic tests in children: review of the literature from 1999 to 2009. Eur J Pediatr. 2010;169(1):15-25. doi: http://dx.doi.org/10.1007/s00431-009-1033-x

5. Kliegman R, Nelson W. Nelson Textbook of Pediatrics. 19a ed. Philadelphia, PA: Elsevier/Saunders; 2011. p. 2610.

6. Husby S, Koletzko S, Korponay-Szabó IR, Mearin ML, Phillips A, Shamir R, et al. European Society for Pediatric Gastroenterology, Hepatology, and Nutrition guidelines for the diagnosis of coeliac disease. J Pediatr Gastroenterol Nutr. 2012;54(1):136-160. doi: http://dx.doi.org/10.1097/ MPG.0b013e31821a23d0

7. Moscoso F, Quera R. Enfermedad celiaca: revision. Rev Med Chile. 2016; 144:211-221.

8. Tack GJ, Verbeek WH, Schreurs MW, Mulder CJ. The spectrum of celiac disease: epidemiology, clinical aspects and treatment. Nat Rev Gastroenterol Hepatol. 2010; 7(4):204-213. doi:http://dx.doi.org/10.1038/nrgastro.2010.23

9. Rostami-Nejad M, Aldulaimi D, Livett H, Rostami K. H.pylori associated with iron deficiency anemia even in celiac disease patients; strongly evidence based but weakly reflected in practice. Gastroenterol Hepatol Bed Bench. 2015;8(3): 178-182.

10. Samasca G, Deleanu D, Sur G, Lupan I, Giulia A, Carpa $R$. Is it necessary to screen Helicobacter pylori infection in patients with celiac disease and iron deficiency? Gastroenterol Hepatol Bed Bench. 2016;9(4):345.

11. Broide E, Sandbank J, Scapa E, Kimchi NA, Shapiro M, Lerner A. The immunohistochemistry profile of lymphocytic gastritis in celiac disease and helicobacter pylori infection: interplay between infection and inflammation. Mediators Inflamm. 2007;2007:81838. doi: http://dx.doi.org/10.1155/2007/81838
12. Medina MG, Medina ML, Martín GT, Dikstein BC, Picón SO, Gorodner JO, et al. Presencia de Helicobacter pylori en biopsias gástricas y heces de pacientes pediátricos con enfermedad celíaca. Rev Gastroenterol Mex. 2009;74(2):94-98.

13. Ciacci C, Squillante A, Rendina D, Limauro S, Bencivenga C, Labanca F, et al. Helicobacter pylori infection and peptic disease in coeliac disease. Eur J Gastroenterol Hepatol. 2000;12(12):1283-7. doi: http://dx.doi.org/10.1097/00042737-200012120-00004

14. Luzza F, Mancuso M, Imeneo M, Mesuraca L, Contaldo A, Giancotti L, et al. Helicobacter pylori infection in children with celiac disease: prevalence and clinicopathologic features. J Pediatr Gastroenterol Nutr. 1999;28(2):143-146. doi: http://dx.doi.org/10.1097/00005176-199902000-00009

15. Aydogdu S, Cakir M, Yuksekkaya HA, Tumgor G, Baran M, Arikan C, et al. Helicobacter pylori infection in children with celiac disease. Scand J Gastroenterol. 2008;43(9):1088-93. doi: http://dx.doi.org/10.1080/00365 520802101846

16. Rostami-Nejad M, Villanacci V, Mashayakhi R, Molaei M, Bassotti G, Zojaji H, et al. Celiac disease and Hp infection association in Iran. Rev Esp Enferm Dig. 2009;101(12):850-854. doi: http://dx.doi.org/10.4321/s113001082009001200004

17. Rostami-Nejad M, Rostami K, Yamaoka Y, Mashayekhi R, Molaei M, Dabiri H, et al. Clinical and histological presentation of Helicobacter pylori and gluten related gastroenteropathy. Arch Iran Med. 2011;14(2):115-118.

18. Zacur M, Duarte D, Petit S, Ibieta F, Nunez M. Helicobacter Pylori en Niños. Pediatr. (Asunción) [Internet]. 2006 [citado 03 Ago. 2020]; 33(1): 26-31. Disponible en: http://scielo.iics.una.py/scielo.php?script= sci_arttext\&pid=S1683-98032006000100005\&lng=es.

19. Villanacci V, Bassotti G, Liserre B, Lanzini A, Lanzarotto F, Genta RM. Helicobacter pylori infection in patients with celiac disease. Am J Gastroenterol. 2006;101(8):1880-1885. doi: http://dx.doi.org/10.1111/ j.1572-0241.2006.00621.x 